\title{
Equal values of pyramidal numbers
}

\author{
Tünde Kovács ${ }^{\mathrm{a}, 1}$, Zsolt Rábai b,2,* \\ ${ }^{a}$ University of Debrecen \\ H-4010 Debrecen, P.O. Box 12, Hungary \\ ${ }^{b}$ MTA-DE Research Group "Equations Functions and Curves" \\ Hungarian Academy of Sciences and University of Debrecen \\ H-4010 Debrecen, P.O. Box 12, Hungary
}

\begin{abstract}
Let $\operatorname{Pyr}_{m}(x)$ denote the pyramidal number with integer parameters $m \geq 3$ and $x \geq 3$. In this note we investigate the Diophantine equation $\operatorname{Pyr}_{m}(x)=\operatorname{Pyr}_{n}(y)$ in positive integer unknowns $x$ and $y$, where $m$ and $n$ are given, different integers. We deduce an effective upper bound for the size of the solutions. Our proof is based on the basic properties of elliptic curves, and elliptic integrals.
\end{abstract}

Keywords: pyramidal numbers, elliptic curves, elliptic logarithms 11D25, 14G05

\section{Introduction}

Let $m$ be a fixed integer with $m \geq 3$. Then the number

$$
\operatorname{Pyr}_{m}(x)=\frac{x(x+1)((m-2) x+5-m)}{6}
$$

is called the pyramidal number with parameters $m$ and $x$. Interesting aspects of pyramidal numbers are the binomial coefficients $\operatorname{Pyr}_{3}(x)=\left(\begin{array}{l}x \\ 3\end{array}\right)$ with integers $x \geq 3$, and the successive partial sum of the series of triangular numbers. According to Dickson [Dic12], the first mention of pyramidal numbers dates back to the ancient Greece. For detailed historical background, please refer to [Dic12]. Pyramidal numbers and their generalizations, figurate numbers, play an important role in discrete mathematics and number theory. (For a detailed introduction into figurate numbers, please consult [DD12].) The diophantine and arithmetic properties of pyramidal and figurate numbers have been widely investigated over the years. Dickson [Dic12] proved, that every sufficiently large integer is the sum of eight pyramidal numbers. Numerical results due to Richmond [Ric44] and Deng and Yang [DY94] make it is plausible that the result of Dickson can be improved.

\footnotetext{
${ }^{*}$ Corresponding author

Email addresses: tkovacsescience. unideb.hu (Tünde Kovács ), zsrabai@science. unideb.hu (Zsolt Rábai )

${ }^{1}$ This research was supported in part by the Japan Society for the Promotion of Science JSPS P12806.

${ }^{2}$ This research was supported in part by the Hungarian Academy of Sciences, and the European Union and the State of Hungary, co-financed by the European Social Fund in the framework of TÁMOP-4.2.4.A/ 2-11/1-2012-0001 'National Excellence Program'.
} 
There are also several classical results related to the equal values of pyramidal and other combinatorial numbers. In 1962, Segal [Seg62] proved, that 10 is the only pyramidal number whose double is also a pyramidal number. In 1998, Brindza, Pintér and Turjányi [BPT98] investigated the equal values of pyramidal and polygonal numbers. They considered the equation

$$
\operatorname{Poly}_{m}(x)=\operatorname{Pyr}_{n}(y)
$$

where Poly ${ }_{m}(x)$ denotes the sequence of polygonal numbers (for details please refer to [BPT98]) and proved that for all but a finite, computable set of pairs $(m, n), \max (x, y)$ is effectively bounded. In 2012, Dujella, Győry and Pintér [DGP12] studied the power values of pyramidal numbers. Recently, in two papers Pintér and Varga [PV11] and Hajdu, Tengely, Pintér and Varga [HPTV14] used various effective methods to investigate the equal values of general figurate numbers.

The purpose of this paper is to study the diophantine equation

$$
\operatorname{Pyr}_{m}(u)=\operatorname{Pyr}_{n}(v)
$$

in positive integers $u$ and $v$ for given $m$ and $n$. In what follows, we give effective upper bounds for the size of the solutions of (2). We apply the so-called Elliptic Logarithm method, which was developed by Stroeker and Tzanakis [ST94], and independently by Gebel, Pethő and Zimmer [GPZ94] and later improved by Stroeker and Tzanakis [ST03]. Two interesting special cases are studied by computational number-theoretic tools. Before stating the main results of the paper, we would like to introduce another form of the problem. It is easy to see that (2) is equivalent to the equation

$$
(m-2) u^{3}+3 u^{2}+(5-m) u=(n-2) v^{3}+3 v^{2}+(5-n) v
$$

in positive integer unknowns $u$ and $v$. With this latter form, the main results of the paper are the following.

Theorem 1.1. Let $m$ and $n$ be given positive integers with $3 \leq \min (m, n)$ and $m \neq n$. Then the equation (3) has at most finitely many solutions in integer unknowns $u$ and $v$. In fact $\max (u, v)<C_{1}$, where $C_{1}$ is an effectively computable positive constant depending only on $m$ and $n$.

Remark We would like to mention here, that Theorem 1.1 is also a direct consequence of the celebrated result of Baker and Coates (see [BC70]). However, the currently discussed Elliptic Logarithm method gives more practical bounds. Sadly, due to the nature of the method, it is currently not possible to make $C_{1}$ explicit in terms of $m$ and $n$.

Using the techniques mentioned above and the program packages MAGMA [BCP97], SAGE [Dev15] and MAPLE, we prove

Theorem 1.2. For given $m$ and $n$ with $3 \leq n<m \leq 10$, all solutions of (3) in $(u, v)$ integers with $(u, v) \notin\{(0,0)(-1,-1),(-1,0),(0,-1),(1,1)\}$ are given in the following table. 


\begin{tabular}{|c|c|}
\hline$(m, n)$ & $(u, v)$ \\
\hline$(4,3)$ & $(0,-2),(-1,-2)$ \\
\hline$(5,3)$ & $(0,-2),(-1,-2),(-35,-51)$ \\
\hline$(6,3)$ & $(0,-2),(-1,-2),(-16,-26)$ \\
\hline$(7,3)$ & $(0,-2),(-1,-2),(-2,-4)$ \\
\hline$(7,5)$ & $(-5,-6),(6,7)$ \\
\hline$(8,3)$ & $(0,-2),(-1,-2),(7,12)$ \\
\hline$(8,4)$ & $(-2,-3),(3,4)$ \\
\hline$(8,6)$ & $(-276,-316)$ \\
\hline$(9,3)$ & $(0,-2),(-1,-2),(-8,-16),(2,3)$ \\
\hline$(9,4)$ & $(-13,-20)$ \\
\hline$(9,7)$ & $(152,170)$ \\
\hline$(10,3)$ & $(0,-2),(-1,-2)$ \\
\hline$(10,4)$ & $(55,87)$ \\
\hline$(10,6)$ & $(35,44)$ \\
\hline
\end{tabular}

As a direct corollary to Theorem 1.2, we can state the following.

Corollary 1. For given $m$ and $n$ with $3 \leq n<m \leq 10$, all solutions of (2) in positive integers $(u, v)$ with $(u, v) \neq(1,1)$ are given by

$$
(m, n, u, v) \in\left\{\begin{array}{c}
(8,3,7,12),(9,3,2,3),(8,4,3,4),(10,4,55,87), \\
(7,5,6,7),(10,6,35,44),(9,7,152,170) .
\end{array}\right\}
$$

Before proceeding with the proofs, we would like to make some preliminary remarks.

Remarks. Easy substitution shows that the elements of the excluded set

$$
\{(0,0),(-1,-1),(-1,0),(0,-1),(1,1)\}
$$

are solutions of (3) for all $m$ and $n$.

As the computational data shows, giving all the solutions with unknown $m, n, u$ and $v$ is hopeless, as there does not seem to be any pattern in the solutions. If we consider (2) for given $u$ and $v$ in integer unknowns $m$ and $n$, we get a linear equation. In this case (2) has either no solutions or infinitely many. The latter is the case if and only if

$$
\operatorname{gcd}\left(u^{3}-u, v^{3}-v\right) \mid\left(2 u^{3}-3 u^{2}-5 u-2 v^{3}+3 v^{2}+5 v\right)
$$

We would also like to mention here, that in the case where both $m-2$ and $n-2$ are perfect cubes, we can apply elementary calculations to deduce an upper bound for $\max \{u, v\}$. Indeed, suppose that in (3), $m-2=k^{3}$ and $n-2=l^{3}$. Set

$$
U=k^{3} l^{2} u+l^{2}, \quad V=k^{2} l^{3} v+k^{2} .
$$

Then we have

$U^{3}-V^{3}=l^{4}\left(\left(k^{3}-3\right) k^{3}+3\right) U-k^{4}\left(\left(l^{3}-3\right) l^{3}+3\right) V+l^{6}\left(3 k^{3}-2\right)-k^{6}\left(3 l^{3}-2\right)$.

Here $U=V$ cannot occur. Using the triangle inequality, we get

$$
(\max \{U, V\})^{2} \leq U^{2}+U V+V^{2} \leq C_{0} \max \{U, V\},
$$


with

$$
C_{0}=k^{4}\left(\left(l^{3}-3\right) l^{3}+3\right)+l^{4}\left(\left(k^{3}-3\right) k^{3}+3\right)+k^{6}\left(3 l^{3}-2\right)+l^{6}\left(3 k^{3}-2\right),
$$

and so

$$
\max \{U, V\} \leq C_{0}
$$

\section{Auxiliary Results}

Lemma 2.1. Let $m$ and $n$ be given positive integers with $m \neq n$. Then equation (3) is birationally equivalent to the Weierstrass curve

$$
y^{2}=x^{3}+c(m, n) x+d(m, n),
$$

where

$$
\begin{aligned}
c(m, n)= & -48 n^{2} m^{2}+336 n m^{2}+ \\
& +4368 m-624 m^{2}-624 n^{2}+ \\
& +4368 n-8112-2352 n m+336 m n^{2},
\end{aligned}
$$

and

$$
\begin{aligned}
d(m, n)= & 281216-227136 m-227136 n-4352 n^{3}-4352 m^{3}+256 m^{4}- \\
& -52544 n m^{2}++57424 n^{2}+57424 m^{2}+194656 n m+64 n^{4} m^{2}- \\
& -52544 m n^{2}+4352 m n^{3}+4352 n m^{3}-256 n^{4} m-256 n m^{4}- \\
& -1088 n^{2} m^{3}+64 n^{2} m^{4}+256 n^{4}+14592 n^{2} m^{2}-1088 n^{3} m^{2} .
\end{aligned}
$$

Moreover, there exist mutually invertible birational transformations $\Phi(x, y)$ and $\Psi(x, y)$, under which

$$
u=\Phi(x, y) \text {, and } v=\Psi(x, y) \text {. }
$$

ProOF. We prove Lemma 2.1 using an algorithm due to Nagell [Nag29]. We will closely follow the method described by Connell in [Con96]. Let us start with (3), which has the rational point $(u, v)=(0,0)$.

Step 1 . Substitute $u=U / W$ and $v=V / W$ in (3), and clear the denominators to get the homogenous form

$$
F=F_{3}+F_{2} W+F_{1} W^{2}=0
$$

where

$$
\begin{aligned}
& F_{3}=(m-2) U^{3}-(n-2) V^{3}, \\
& F_{2}=3 U^{2}-3 V^{2}, \\
& F_{1}=(5-m) U-(5-n) V .
\end{aligned}
$$

The rational point $P$ with $(u, v)=(0,0)$ has the projective coordinates $[U: V$ : $W]=[0: 0: 1]$. The tangent line to $(8)$ in $P$, given by $F_{1}=0$ meets $(8)$ in $Q=$ $\left[e_{2}(5-n), e_{2}(5-m), e_{3}\right]$, where

$$
\begin{aligned}
& e_{2}=F_{2}(-(5-n),-(5-m))=3(5-n)^{2}-3(5-m)^{2}, \\
& e_{3}=F_{3}(-(5-n),-(5-m))=-(m-2)(5-n)^{3}+(n-2)(5-m)^{3} .
\end{aligned}
$$

The aim of this step is to bring $Q$ into the origin with a suitable change of coordinates. Before we can do this, we have to examine $e_{2}$ and $e_{3}$ a little further. It is easy to see, 
that $e_{2}$ can only be 0 if and only if $|5-m|=|5-n|$, which means that $(m, n)$ comes from the set

$$
S=\{(1,9),(2,8),(3,7),(4,6),(6,4),(7,3),(8,2),(9,1)\} .
$$

On the other hand, $e_{3}$ cannot be 0 , since that would mean, that

$$
\frac{(5-m)^{3}}{m-2}=\frac{(5-n)^{3}}{n-2}
$$

holds. But the derivative of $\frac{(5-x)^{3}}{x-2}$ is negative for every positive integer $x$ other than 5 . Thus, for $x \neq 5$, the function

$$
\frac{(5-x)^{3}}{x-2}
$$

is monotonous which means, that for $m \neq n \neq 5$, (9) cannot occur. By choosing $m=5$ in (9), we get, that $n=5$, which contradicts $m \neq n$.

Thus we can distinguish two cases: either we have $e_{2} \neq 0$ and $e_{3} \neq 0$ with $(m, n) \notin S$, or we have $e_{2}=0, e_{3} \neq 0$ with $(m, n) \in S$. If $(m, n)$ is in $S$, then $Q$ is the origin, thus $Q$ is a flex. If this is the case, one can jump directly to Step 2 .

We make the coordinate transformation

$$
\begin{aligned}
& U=U^{\prime}+\frac{(5-n) e_{2}}{e_{3}} W^{\prime}, \\
& V=V^{\prime}+\frac{(5-m) e_{2}}{e_{3}} W^{\prime}, \\
& W=W^{\prime}
\end{aligned}
$$

to send $Q$ to the origin. We can now return to affine coordinates $u^{\prime}=U^{\prime} / W^{\prime}, v^{\prime}=$ $V^{\prime} / W^{\prime}$.

Step 2. Now our equation is of the form $f^{\prime}=f_{1}^{\prime}+f_{2}^{\prime}+f_{3}^{\prime}=0$, where $f_{i}^{\prime}$ denotes the homogenous part of $f^{\prime}\left(u^{\prime}, v^{\prime}\right)$ of degree $i, i \in\{1,2,3\}$. Introduce $t=\frac{v^{\prime}}{u^{\prime}}$, and denote $f_{i}^{\prime}(1, t)$ by $\phi_{i}$ to get the quadratic equation

$$
u^{\prime 2} \phi_{3}+u^{\prime} \phi_{2}+\phi_{1}=0
$$

Let $\delta=\phi_{2}^{2}-4 \phi_{3} \phi_{1}$. Then

$$
u^{\prime}=\frac{-\phi_{2} \pm \sqrt{\delta}}{2 \phi_{3}}, \text { and } v^{\prime}=t u^{\prime}
$$

The zeros of $\delta$ are the slopes of the tangents to the curve in the $\left(u^{\prime}, v^{\prime}\right)$-plane that pass through $Q$. One such value is

$$
t_{0}=\frac{5-m}{5-n} .
$$

Thus $\left(t-t_{0}\right)$ is a linear factor of $\delta$. Write $\tau=\left(t-t_{0}\right)^{-1}$, and let $\rho=\tau^{4} \delta$. Clearly, $\rho$ is a cubic polynomial in $\tau$.

Step 3. Finally, if

$$
\rho=c_{1} \tau^{3}+c_{2} \tau^{2}+c_{3} \tau+c_{4},
$$

then substitute $\tau=\frac{x^{\prime}}{c_{1}}, \rho=\frac{y^{2}}{c_{1}^{2}}$ to get the elliptic equation

$$
y^{2}=x^{\prime 3}+c_{2} x^{\prime 2}+c_{1} c_{3} x^{\prime}+c_{1}^{2} c_{4} .
$$

Substituting $x^{\prime}=x-\frac{c_{2}}{3}$ yields (6). The transformations $\Phi$ and $\Psi$ can be traced back starting with (11). 


\section{Outline of the proof of Theorem 1.1}

By Lemma 2.1, our initial equation is equivalent to the Weierstrass curve (6). Our goal is to apply the Elliptic Logarithm method to deduce the upper bound $c_{1}$. To do this, we have to make sure first, that (6) is non-singular. The discriminant of (6) is

$$
D(m, n)=-6912 \cdot D_{1}(m, n) \cdot(m-n)^{2}
$$

where

$$
\begin{aligned}
& \quad D_{1}(m, n)= \\
& -12303200-4987111 n^{2}-4987111 m^{2}-14047282 n m+4409272 n m^{2}+ \\
& +4409272 m n^{2}-90912 m n^{3}-90912 n m^{3}+13540280 m+13540280 n+546080 n^{3}+ \\
& +546080 m^{3}+48224 m^{4}-8704 m^{5}+256 m^{6}+48224 n^{4}-8704 n^{5}+256 n^{6}- \\
& -637744 n^{2} m^{2}-436552 n^{3} m^{2}-158176 n^{4} m+154792 n^{4} m^{2}-158176 n m^{4}- \\
& -436552 n^{2} m^{3}+154792 n^{2} m^{4}+17920 n^{5} m-14080 n^{5} m^{2}+287712 n^{3} m^{3} \\
& -65376 n^{3} m^{4}-512 n^{6} m+384 n^{6} m^{2}-65376 n^{4} m^{3}+12176 n^{4} m^{4}+17920 m^{5} n \\
& -14080 m^{5} n^{2}-512 m^{6} n+384 m^{6} n^{2}+5120 n^{5} m^{3}-800 n^{5} m^{4}+5120 n^{3} m^{5}- \\
& -128 n^{3} m^{6}-128 n^{6} m^{3}-800 n^{4} m^{5}+32 n^{5} m^{5}+16 m^{4} n^{6}+16 m^{6} n^{4} .
\end{aligned}
$$

Clearly, $D(m, n)=0$ if and only if $D_{1}(m, n)=0$. Write

$$
\begin{aligned}
\text { Pol }_{0}= & 256 n^{6}-8704 n^{5}+48224 n^{4}+546080 n^{3}-4987111 n^{2}+13540280 n- \\
& -12303200, \\
\text { Pol }_{1}= & -512 n^{6}+17920 n^{5}-158176 n^{4}-90912 n^{3}+4409272 n^{2}-14047282 n+ \\
& +13540280+m\left(384 n^{6}-14080 n^{5}+154792 n^{4}-436552 n^{3}-637744 n^{2}+\right. \\
& +4409272 n-4987111), \\
\text { Pol }_{2}= & -128 n^{6}+5120 n^{5}-65376 n^{4}+287712 n^{3}-436552 n^{2}-90912 n+546080+ \\
& +m\left(16 n^{6}-800 n^{5}+12176 n^{4}-65376 n^{3}+154792 n^{2}-158176 n+48224\right), \\
\text { Pol }_{3}= & 32 n^{5}-800 n^{4}+5120 n^{3}-14080 n^{2}+17920 n-8704, \\
\text { Pol }_{4}= & 16 n^{4}-128 n^{3}+384 n^{2}-512 n+256 .
\end{aligned}
$$

Thus, $D_{1}=\mathrm{Pol}_{4} m^{6}+\mathrm{Pol}_{3} m^{5}+\mathrm{Pol}_{2} m^{3}+\mathrm{Pol}_{1} m+\mathrm{Pol}_{0}$. It is obvious, that for suitably large $m$ all the polynomials $\mathrm{Pol}_{i}$ are monotonously increasing in $n$, thus always positive for large enough $n$. Easy calculation shows that $D_{1}>0$ for $\min \{m, n\}>30$. Now fix $m$ for $m=m_{0} \leq 30$. Then $D_{1}\left(m_{0}, n\right)$ is a polynomial in $n$. Searching for the integer roots of $D_{1}\left(m_{0}, n\right)$ for all $1 \leq m_{0}<30$ we find, that $D_{1}(m, n)=0$ can occur only with $m=n=5$. Repeating this last step with fixing $n=n_{0} \leq 30$ we get the same result thus proving that $(6)$ is non-singular for all $(m, n)$, where $m \neq n$.

Now, we turn to the Elliptic Logarithms. Here we follow the approach described by Stroeker and Tzanakis in [ST94] and Stroeker de Weger in [SdW98]. Let $r$ be the rank of the curve (6), $P_{1}, \ldots P_{r}$ a basis of the Mordell-Weil group, and $P_{r+1}$ a torsion pont on $E$. Then a rational point $P$ on the curve is of the shape $P=m_{1} P_{1}+\cdots+$ $m_{r} P_{r}+P_{r+1}$ with $m_{i} \in \mathbb{Z}$. Write $M=\max _{1 \leq i \leq r}\left|m_{i}\right|$. Then according to [SdW98] the linear form in elliptic logarithms has the form

$$
L(P)=m_{0} \omega+m_{1} u_{1}+\cdots+m_{r} u_{r}+u_{r+1}-u_{0},
$$

where $u_{i}$ are the elliptic logarithms of the points $P_{i}, u_{0}$ is the elliptic logarithm of a well-chosen $Q_{0}$ point on E, and $m_{0}$ is a scaling factor. Using this notation, we have 
$\max \left\{M,\left|m_{0}\right|\right\} \leq r M+1$.

On one hand, we have an upper bound for this linear form:

$$
|L(P)|<\exp \left(c_{1}-c_{2} M^{2}\right),
$$

where the constants $c_{1}$ and $c_{2}$ are effectively determinable. On the other hand a result by David [Dav95] provides a lower bound for the linear form $L(P)$. Combining this with (13) provides an upper bound for $M$. Thus $x$ and $y$ are bounded in terms of $m$ and $n$, which combined with (7) yields an upper bound for $\max \{u, v\}$ completing the proof of Theorem 1.1.

\section{Examples}

In what follows, we will illustrate the method described in Section 3 in two interesting special cases. Iterating the steps described here for $3 \leq n \leq m \leq 10$, we get the set of solutions (4), thus proving Theorem 1.2. First, we consider (3) with $(m, n)=(9,7)$. More precisely, we prove the following theorem.

Theorem 4.1. The only positive integer, which is both 9-pyramidal and 7-pyramidal, is 4108560 .

Theorem 4.1 is the direct consequence of the following lemma.

Lemma 4.1. All solutions of the equation

$$
7 u^{3}+3 u^{2}-4 u=5 v^{3}+3 v^{2}-2 v
$$

in integer unknowns $u$ and $v$ are

$$
(u, v) \in\{(-1,-1),(-1,0),(0,-1),(1,1),(152,170)\} .
$$

Proof. Equation (14) is birationally equivalent to the minimal Weierstrass curve

$$
Y^{2}=X^{3}-1209 X+19361,
$$

under the transformation

$$
(X, Y)=\left(\frac{20 u-18-43 v}{2 u-v}, \frac{3\left(231 u^{3}+129 u^{2}-26 u-165 v^{3}-156 v^{2}-14 v\right)}{(2 u-v)^{2}}\right) .
$$

Using the program package MAGMA, we get that the rank of (16) is 4, and the torsion subgroup of $(16)$ is $\mathcal{O}$. The generators of the Mordell-Weil group are:

$$
P_{1}=(19,57), P_{2}=(25,-69), P_{3}=(-5,-159), P_{4}=(-41,3) .
$$

Let $P=m_{1} P_{1}+m_{2} P_{2}+m_{3} P_{3}+m_{4} P_{4}$ be a rational point on (16) which is the image of an integer point on (14). Then the linear form $L(P)$ is of the shape

$$
L(P)=m_{0} \omega+m_{1} u_{1}+m_{2} u_{2}+m_{3} u_{3}+m_{4} u_{4}-u_{0},
$$

where $\omega$ is the fundamental real period of $(16)$, and $u_{i}(i=0, \ldots, 4)$ are the elliptic logarithms of $Q_{0}$ and $P_{i},(i=1, \ldots, 4)$. After some calculation we have

$$
Q_{0}=(-31.8884 \ldots, 159.6485 \ldots),
$$




$$
\begin{aligned}
\omega & =2.1510 \ldots, u_{0}=0.9728 \ldots, u_{1}=0.5717 \ldots, \\
u_{2} & =1.6797 \ldots, u_{3}=1.3289 \ldots, u_{4}=1.0739 \ldots
\end{aligned}
$$

Also, in this particular case, (13) reads as

$$
|L(P)|<\exp \left(10.168-1.23 M^{2}\right) .
$$

Combining this with the aforementioned result of David, we get $M<0.384 \cdot 10^{116}$. To reduce the upper bound, we apply de Weger's [dW89] method based on the $L L L$ algorithm. After a few iterations we get $M<13$. Searching for all points on (16) with $M<13$, and applying the inverse of the birational transformations mentioned above, we are able to calculate the set of solutions (15).

As a second application for the method, we consider the following problem. For given integers $x \geq 1$ and $y \geq 1$, what are the solutions of the diophantine equation

$$
\left(\begin{array}{c}
x+2 \\
3
\end{array}\right)=1^{2}+2^{2}+\cdots+y^{2} .
$$

Using the definition of the binomial coefficients, and some well-known properties of sums of squares, we get, that (17) is equivalent to (3) with $(m, n)=(4,3)$. We have the following theorem.

Theorem 4.2. The only solution of (17) in integers $x \geq 1$ and $y \geq 1$ is the trivial solution $(x, y)=(1,1)$.

This is the direct consequence of the following lemma.

Lemma 4.2. The diophantine equation

$$
2 u^{3}+3 u^{2}+u=v^{3}+3 v^{2}+2 v
$$

has no solutions in integers $(u, v)$ other than the trivial solutions $(u, v) \in\{(-1,-1),(-1,0),(0,-1)(1,1)\}$.

PROOF. We proceed as in the previous case. Equation (18) is birationally equivalent to the minimal Weierstrass curve

$$
Y^{2}=X^{3}-48 X+272
$$

under the transformation

$$
(X, Y)=\left(\frac{-4(5 u+9+5 v)}{u-2 v}, \frac{12\left(30 u^{3}+66 u^{2}+41 u-15 v^{3}-39 v^{2}-28 v\right)}{(u-2 v)^{2}}\right) .
$$

The rank of (19) is 1 , and the torsion subgroup of (19) is trivial. The generator of the Mordell-Weil group is

$$
P_{1}=(16,60) \text {. }
$$

Denote by $P=m_{1} P_{1}$ a rational point on (19), which is the image of an integer solution of (18). Then we have the following linear form

$$
L(P)=m_{0} \omega+m_{1} u_{1}-u_{0},
$$


where $\omega$ is the fundamental real period of (18), and $u_{0}, u_{1}$ are the elliptic logarithms of the points $P_{1}$ and $Q_{0}=(29.7388 \ldots, 158.5738 \ldots)$. After some calculation, we have

$$
\omega=3.7814 \ldots, u_{0}=0.3685 \ldots, u_{1}=0.5074 \ldots
$$

In this case, (13) reads as

$$
|L(P)|<\exp \left(8.02852-0.05909 M^{2}\right) .
$$

Combining this with David's result, we get $M<0.2919 \cdot 10^{52}$. After a few iterations of de Weger's algorithm, we arrive at $M<18$. Checking for solutions with this condition, we find that the only solution of (18) is the trivial one.

\section{Computational Remarks}

At the request of the referee, we would like to make some remarks concerning the practical details of the computation. Due to MAPLE's powerful symbolic computational capabilities, the birational transformation between (3) and the Weierstrass model was computed using a simple implementation of Nagell's algorithm in MAPLE 18. The calculation of the elliptic logarithms, the upper bound and the reduction was also done in MAPLE 18, using MAPLE's built-in LLL routine. The computation of the group of rational points on the curves and the search using the bounds from the reduction were carried out in both MAGMA and SAGE. The runtimes on a personal computer equipped with an AMD A10-7800 CPU ranged from being only a few seconds to few minutes depending on the curves. The most time consuming steps were the computation of the Mordell-Weil generators, the reduction process and the exhaustive search below the reduced bound. Compared to the run times presented for example in [SdW99], no big difference is seen. This may be caused by the small absolute value of the parameters. The goal of the present paper is investigate an interesting family of diophantine equations, as the run times show however one can extend these results to higher values of $m$ and $n$ if desired. Also, we think that an algorithm could be written in MAGMA (or any other of the three), that gives a list of all solutions for given $m$ and $n$, similarly to some other special cases of genus 1 equation solvers already present in MAGMA.

Acknowledgements. The authors would like to express their thanks to Ákos Pintér and the anonymous referee for their helpful comments.

[BC70] A. Baker and J. Coates. Integer points on curves of genus 1. Mathematical Proceedings of the Cambridge Philosophical Society, 67(3):595-602, 1970.

[BCP97] W. Bosma, J. Cannon, and C. Playoust. The magma algebra system I: The user language. J. Symbolic Comput., 24(3):235-265, 1997.

[BPT98] B. Brindza, Á. Pintér, and S. Turjányi. On equal values of pyramidal and polygonal numbers. Indag. Math. (N.S.), 9(2):183-185, 1998.

[Con96] I. Connell. Elliptic curve handbook. Preprint, 1996. 
[Dav95] S. David. Minorations de formes linéaires de logarithmes elliptiques. Mémoires de la Societé Mathématique de France, 62:1-143, 1995.

[DD12] E. Deza and M. Deza. Figurate numbers. World Scientific, 2012.

[Dev15] The Sage Developers. Sage Mathematics Software (Version 6.4.1), 2015. http: //www. sagemath.org.

[DGP12] Andrej Dujella, Kálmán Győry, and Ákos Pintér. On power values of pyramidal numbers, I. Acta Arith., 155(2):217-226, 2012.

[Dic12] L. E. Dickson. History of the Theory of Numbers, Volume II: Diophantine Analysis. Courier Dover Publications, 2012.

[dW89] B. M. M. de Weger. Algorithms for Diophantine equations. CWI-Tract No. 65, 1989.

[DY94] Yue-Fan Deng and Chen Ning Yang. Waring's problem for pyramidal numbers. Sci. China Ser. A, 37(3):277-283, 1994.

[GPZ94] J. Gebel, A. Pethô, and H. G. Zimmer. Computing integral points on elliptic curves. Acta Arith., 68(2):171-192, 1994.

[HPTV14] Lajos Hajdu, Ákos Pintér, Szabolcs Tengely, and Nóra Varga. Equal values of figurate numbers. J. Number Th., 137:130-141, 2014.

[Nag29] T. Nagell. Sur les propriétés arithmétiques des cubiques planes du premier genre. Acta Math., 52(1):93-126, 1929.

[PV11] Á. Pintér and N. Varga. Resolution of a nontrivial diophantine equation without reduction methods. Publ. Math. Debrecen, 79(3-4):605-610, 2011.

[Ric44] H. W. Richmond. Notes on a problem of the "Waring" type. J. London Math. Soc., 19:38-41, 1944.

[SdW98] R. J. Stroeker and B. M. M. de Weger. Solving elliptic diophantine equations: the general cubic case. Acta Arith., 87:339-365, 1998.

[SdW99] Roelof Stroeker and Benjamin de Weger. Elliptic binomial diophantine equations. Math. Comput. Amer. Math. Soc., 68(227):1257-1281, 1999.

[Seg62] S. L. Segal. Mathematical Notes: A Note on Pyramidal Numbers. Amer. Math. Monthly, 69(7):637-638, 1962.

[ST94] R. J. Stroeker and N. Tzanakis. Solving elliptic diophantine equations by estimating linear forms in elliptic logarithms. Acta Arith., 67(2):177-196, 1994.

[ST03] R. Stroeker and N. Tzanakis. Computing all integer solutions of a genus 1 equation. Math. Comput. Amer. Math. Soc., 72(244):1917-1933, 2003. 